\title{
Assessment of Isolator Pseudo-shocks Created by Combustion with Heated Flow
}

\author{
Matthew L. Fotia* \& James F. Driscoll ${ }^{\dagger}$ \\ University of Michigan, Ann Arbor, MI 48109, U.S.A.
}

\begin{abstract}
Isolator/combustor interactions are measured in a direct-connect dual-mode ramjet-scramjet experiment. An operating point approach is used to create a mapping of the coupling effects between the isolator geometry, inlet flow conditions and fuel injector behavior. The resulting isolator/injector coupling map provides a description of the response of the isolator to particular injector performance, and the effective blockage it induces on the isolator flow. Existing models and correlations predicting the pressure rise across a pseudo-shock, and its resultant length, were evaluated through comparison with measurements made in a heated-flow isolator duct, coupled to a hydrogen-air combustor. The observation of a normal-to-oblique shock-train transition mechanism has lead to the development of a revised shock-train operating regime description that takes into account the impact of Mach number and maximum pressure recovery on the shock configurations present in the isolator.
\end{abstract}

\section{Nomenclature}

$C_{p} \quad$ Specific Heat at Constant Pressure

$D \quad$ Hydraulic Diameter of Duct

$H \quad$ Duct Height

$h \quad$ Duct Half-height

$M \quad$ Mach Number

$\dot{m} \quad$ Mass Flow-rate

$P \quad$ Pressure

$r \quad$ Fuel-jet Momentum Ratio

$R e_{\theta} \quad$ Reynolds Number based on Boundary-Layer Momentum Thickness

$T$ Temperature

$u \quad$ Flow Velocity

$w \quad$ Crocco Number $\left(=u / \sqrt{2 C_{p} T_{o}}\right)$

$w^{*} \quad$ Crocco Number at Sonic Condition $(=\sqrt{(\gamma-1) /(\gamma+1)})$

${ }^{*}$ Graduate Research Assistant, Department of Aerospace Engineering, AIAA Member, mfotia@umich. edu

${ }^{\dagger}$ Professor, Department of Aerospace Engineering, AIAA Fellow, jamesfd@umich. edu 
$x \quad$ Distance from Pseudo-shock Entrance

$\delta \quad$ Boundary-layer Thickness

$\delta^{*} \quad$ Boundary-layer Displacement Thickness

$\gamma \quad$ Ratio of Specific Heats

$\phi \quad$ Equivalence Ratio of Hydrogen and Oxygen

$\theta \quad$ Boundary-Layer Momentum Thickness

\section{Subscript}

1 Pseudo-shock Entrance Condition

2 Pseudo-shock Exit Condition

e External Flow

$F \quad$ Fuel-jet

$I \quad$ Isolator Flow

$o \quad$ Stagnation Value

$u \quad$ Velocity Value

Superscript

' Supersonic Core Value

\section{Introduction}

Interest in the formation and behavior of shock-trains, and the pseudo-shock phenomena, has been persistent since the mid-1950's. As can be imagined, there are many instances in which a supersonic flow may become confined within a duct. Some examples are supersonic wind-tunnel diffusers and aircraft engine intakes.

However, much of the previous work has been compiled using a room temperature compressed gas sources as a supply to the ducted flows being investigated. Mechanical valves are also used to create the back-pressure that produces the shock-trains. Matsuo et $\mathrm{al}^{1}$ provides a comprehensive review of many of these works. For most of the potential applications this approach is suitable to accurately recreate the behavior of the pseudo-shock, defined as the complete shock-train and

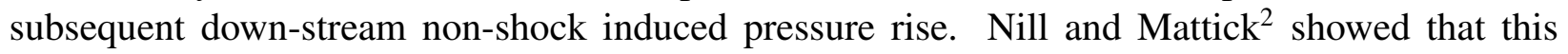
was the case in their experiments involving a shock wave reactor for the purpose of chemical processing.

The flow through the isolator section of a ramjet engine is pre-heated, due to the deceleration of the high-speed external air flow. Any shock-trains present are created by the presence of a downstream combustion process in the engine's combustor section. The work presented here examines the coupling between the combustor and isolator sections in such devices. To provide an adequate description of this coupled behavior, a new concept is proposed that explains the back pressure in terms of a fluid-mechanical blockage and a combustion-induced blockage. It has been found that by making this distinction an operating point approach can be used to describe the behavior of the interdependent isolator/combustor system. Assessments are made to determine the applicability of pseudo-shock models to the heated combustion induced case specific to the ramjet application.

Bement et $\mathrm{al}^{3}$ have previously studied the operation of an isolator with a downstream combustor at a stagnation temperature of $1028 \mathrm{~K}$ and duct flow Mach number of 2.2. A Pitot rake was used to characterize the flow profile across the isolator section. They found that their results compared 
favorably to those estimated by the experimental correlation offered by Waltrup and Billig. ${ }^{4,5}$ Experimental studies have also been presenting by Le et al. ${ }^{6}$ The current work focuses on studying flows at a higher stagnation temperature then Bement et al, as well as offering insight into the actual flow structure of the shock-trains.

The heated flow creates a challenging environment in which to characterize the behavior of such a complex and dynamic flow field. Optical access to the complete test-section can be limited by structural concerns and the difficulties associated with the use of seeding materials, as with techniques such as laser Dopper velocimetry, can be amplified or even rule-out their use entirely.

The influence of isolator geometry on the behavior of the pseudo-shock has been difficult to quantify. The coupling between the fuel injector operation and the response of the isolator is a key relationship that must be explored to better understand the influence of inlet conditions, isolator geometry and fuel injector design on the global operating point performance of a system.

\section{Experimental Setup}

The University of Michigan Dual-Mode Combustor(MDMC) experiment was used to obtain the current set of results. The experiment allows for the examination of the internal flows of a supersonic isolator coupled to a cavity-stabilized combustor, with wall-normal fuel injection. The inlet air can be heated to stagnation temperatures between $1050-1450 \mathrm{~K}$ through $\mathrm{H}_{2}-\mathrm{O}_{2}$ combustion, entering the isolator section of the experiment at a Mach number of 2.2. This enables the experiment to operate under both ram- and scram-jet combustion modes with equivalence ratios between $0-0.42$. A schematic of the experiment can be found in Figure 1.

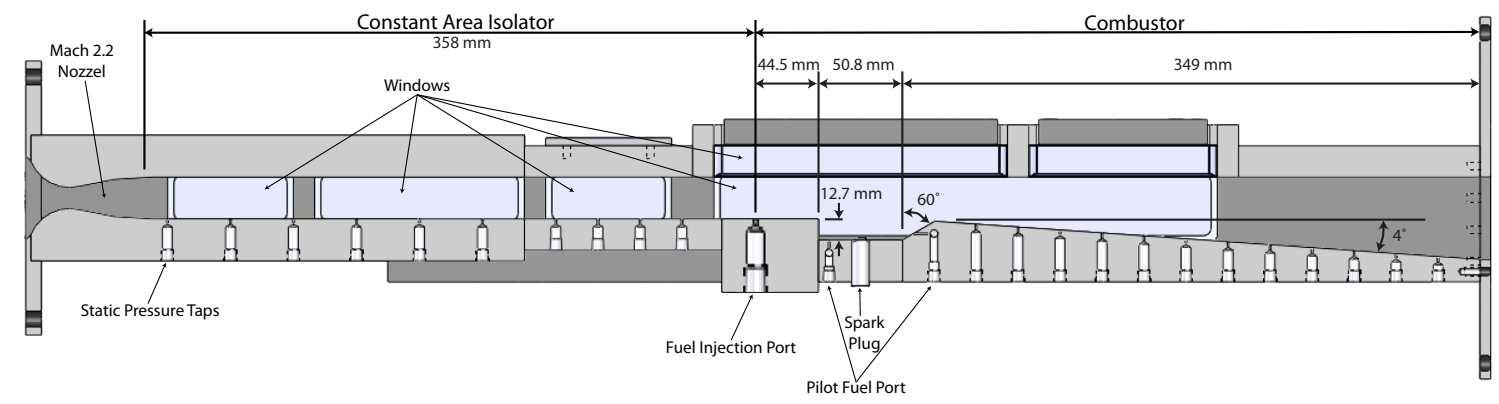

Figure 1. Schematic of the University of Michigan dual-mode combustor experiment.

The MDMC is constructed of stainless steal and inconel alloys. The pre-combustion section of the experiment consisting of an planar inlet nozzle to accelerate the inlet gases and a constant area isolator of $25.4 \mathrm{~mm}$ (height) x $38.1 \mathrm{~mm}$ (width) cross-section. The combustor section includes a wall-normal fuel injector, cavity flame-holder with angled rear-wall, and a $4^{\circ}$ diverging combustor exit. The fuel injector is of a choked-orifice type, providing injection at sonic conditions. The experiment is cooled through natural convection with the laboratory air, and is operated for a sufficient length for time to allow for the establishment of steady flow behavior, but not the degradation of the materials used in the construction of the experiment.

A total of 22 pressure-tap ports are located along the length of the test-section, instrumented with transducers providing error of $0.25 \%$ full scale, or $\pm 0.9 \mathrm{kPa}$, sampled at $40 \mathrm{~Hz}$ using a National Instruments PCI-6229 data acquisition card. All other pressures were recorded at similar, 
or higher, accuracy. The experiment is fully controlled and monitored by computer.

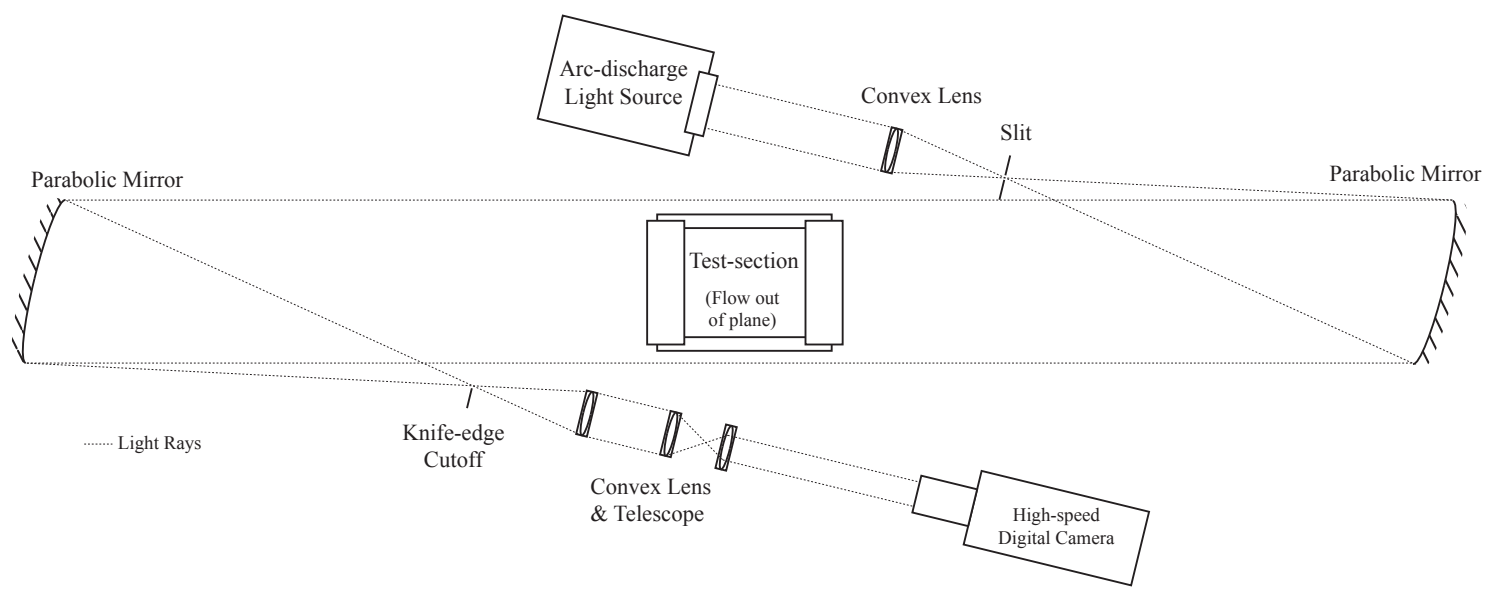

Figure 2. Schematic of the Schlieren imaging apparatus.

Under ramjet operating conditions a shock-train forms in the isolator section due to the back pressure created by the impinging fuel jet and its subsequent combustion. Only gaseous hydrogen fuel was used for the present work.

The behavior of the Michigan Dual-Mode Combustor experiment was detailed by Micka \& Driscoll, ${ }^{7}$ in which the preferred mode of flame stabilization in this particular configuration was examined in detail. The results presented here will consist of pressure transducer measurements alone the length of the isolator section, paired with simultaneous Schlieren imagery of the shocktrains present.

The Schlieren images were captured at $100 \mathrm{~Hz}$ using a Phantom v9.1 high-speed digital camera, with a white arc-discharge light source. The experimental arrangement of the Schlieren system is given in Figure 2. For all of the images presented, the knife-edge has been oriented parallel to the flow inside the isolator to allow the wall-normal flow structure to be examined.

A number of operational conditions were examined. Those cases that will be individually high-lighted later are given in Table 1 for the reader.

\section{The Steady Shock-train}

One of the most informative observations that can be made from the operation of the testsection is the profile of the static pressure inside the device, along its front-to-rear axis. These measurements provide basic information about the pseudo-shock, such as the total pseudo-shock length and maximum isolator pressure rise. However, they also provide more subtle information about the blockage creating the pseudo-shock itself.

To this point, the blockage creating the isolator back-pressure has been near-universally thought of as the equivalent of a simple valve, with many shock-train studies being undertaken with this physical arrangement as a substitute for a combustion-induced back pressure. Figure 3a) shows six experimental conditions, with the same up-stream stagnation pressure but three different stagnation temperatures, $T_{o}=1000,1200 \& 1400 \mathrm{~K}$, and two equivalence ratios, $\phi=0.25 \& 0.30$. The 
Table 1. Selected Experimental Case Conditions

\begin{tabular}{cccccccc} 
Case & $M_{1}$ & $P_{o}$ & $T_{o}$ & $\phi$ & $R e_{\theta}$ & $\theta$ & $r$ \\
\hline & & $\mathrm{kPa}$ & $\mathrm{K}$ & & $10^{3}$ & $\mathrm{~mm}$ & \\
\hline $\mathrm{A}$ & 2.0 & 310.3 & 1000 & 0.30 & 2.50 & $0.248 \pm 0.014$ & 1.452 \\
$\mathrm{~B}$ & 2.0 & 310.3 & 1000 & 0.25 & 2.66 & $0.247 \pm 0.015$ & 1.354 \\
$\mathrm{C}$ & 2.0 & 310.3 & 1200 & 0.30 & 1.79 & $0.198 \pm 0.005$ & 1.417 \\
$\mathrm{D}$ & 2.0 & 310.3 & 1200 & 0.25 & 1.83 & $0.198 \pm 0.004$ & 1.342 \\
$\mathrm{E}$ & 2.0 & 310.3 & 1400 & 0.30 & 1.11 & $0.128 \pm 0.018$ & 1.374 \\
$\mathrm{~F}$ & 2.0 & 310.3 & 1400 & 0.25 & 0.92 & $0.120 \pm 0.023$ & 1.331 \\
$\mathrm{G}$ & 1.9 & 448.2 & 1000 & 0.30 & 2.15 & $0.191 \pm 0.050$ & 1.519 \\
$\mathrm{H}$ & 1.9 & 448.2 & 1000 & 0.25 & 2.10 & $0.190 \pm 0.050$ & 1.436 \\
$\mathrm{I}$ & 1.9 & 448.2 & 1200 & 0.30 & 1.72 & $0.162 \pm 0.024$ & 1.476 \\
$\mathrm{~J}$ & 1.9 & 448.2 & 1200 & 0.25 & 1.61 & $0.170 \pm 0.020$ & 1.400 \\
$\mathrm{~K}$ & 1.9 & 448.2 & 1400 & 0.30 & 0.62 & $0.074 \pm 0.040$ & 1.440 \\
$\mathrm{~L}$ & 1.9 & 448.2 & 1400 & 0.25 & 0.61 & $0.073 \pm 0.040$ & 1.367 \\
$\mathrm{M}$ & 1.82 & 586.1 & 1200 & 0.30 & 0.96 & $0.082 \pm 0.054$ & 1.510 \\
$\mathrm{~N}-\mathrm{W}$ & 1.95 & 379.2 & 1200 & $0.198-0.301$ & $1.73-2.02$ & $0.186-0.189 \pm 0.012$ & $1.183-1.486$
\end{tabular}

equivalence ratio is defined for this investigation as

$$
\phi=\frac{\dot{m}_{F}}{f_{s} \dot{m}_{I, O_{2}}}=\frac{\dot{m}_{F}}{f_{s} Y_{O_{2}} \dot{m}_{I}},
$$

where $f_{s}$ is the stoichiometric fuel-oxidizer ratio. This definition is used to allow for the water vapor component of the inlet air, produced in the $\mathrm{H}_{2}-\mathrm{O}_{2}$ vitiator, to be taken into account. The stagnation temperature change between Cases A, C \& E effectively lowers the mechanical blockage in the combustor, allowing the flow to transition from subsonic(ram) to supersonic(scram) operation. This can be attributed to a resultant increase in the momentum flux through the isolator, relative to that of the wall-normal fuel jet, due to an increase in the velocity of the isolator flow which also offsets the reduction in gas density due to static temperature increase.

The mechanical blockage is defined as the component of the isolator back pressure created by the fluid-mechanical structure of the fuel-jet alone and is described by the momentum flux ratio, formally defined as

$$
r=\left(\frac{\rho_{F} u_{F}^{2}}{\rho_{I} U_{I}^{2}}\right)^{1 / 2}=\left(\frac{\rho_{F} u_{F}^{2}}{\gamma P_{1} M_{1}^{2}}\right)^{1 / 2},
$$

where the relation $\rho u^{2}=(P / R T) u^{2}=(P / \gamma R T) \gamma u^{2}=\gamma P M^{2}$ has been used. The isolator flow quantities in this definition will be evaluated at the pre-pseudo-shock condition, denoted by a subscript 1, to provided the momentum ratio between the obstructing fuel jet and the unobstructed cross-flow. This definition is the supersonic analog to the subsonic instance where the cross-flow quantities are measured external to the influence of the impinging jet.

This mode transition is also seen in Cases B, D \& F, for a lower equivalence ratio, while it should be pointed-out that the transition between Cases $\mathrm{C} \& \mathrm{D}$ is due to a drop in equivalence 
ratio, and its associated momentum ratio change, representing a variation in both mechanical and combustion blockage. The combustion blockage is defined as the component of the isolator back pressure created by the chemical reaction of the fuel and oxidizer, the structure of the induced flame front and the expansion of the downstream produces.

A lower equivalence ratio is coupled to a lower value of momentum ratio as less fuel is being injected into the combustor. However, as can be seen from the ram-mode operation of both Cases B \& D, where one would expect both cases to operate in scram-mode based on the behavior of Case $\mathrm{C}$ at a higher equivalence ratio, the interaction of the mechanical blockage with the combustion induced blockage can have unexpected results. The obstruction present in the combustor does indeed operation like a valve, however one in which there are two coupled components. The relationship between the mechanical and combustion blockage can be either reinforcing, as with the mode transition between Cases C \& D, or destructive, as with the lack of transition between Cases B \& D. The fixed combustor geometry is thought to play a role in influencing the effectiveness of the mechanical blockage, provided by an increase in momentum ratio, relative to that of the combustion induced blockage, provided by an increase in equivalence ratio. A discussion of the influence of isolator geometry on the pressure recovery is provided later in Section IV.

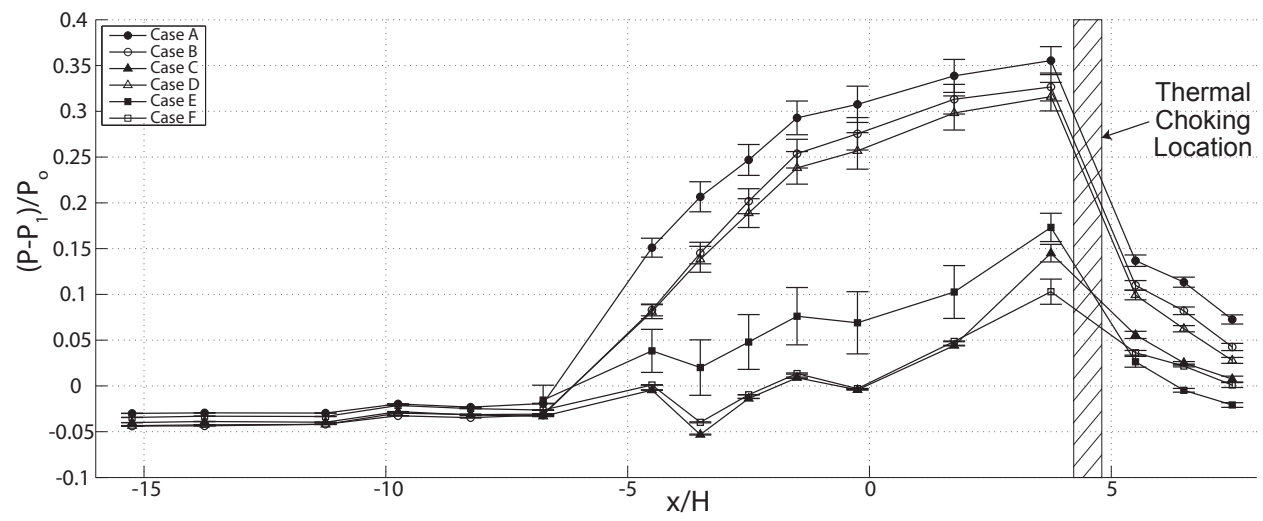

a) Ram- and Scram-mode combustion

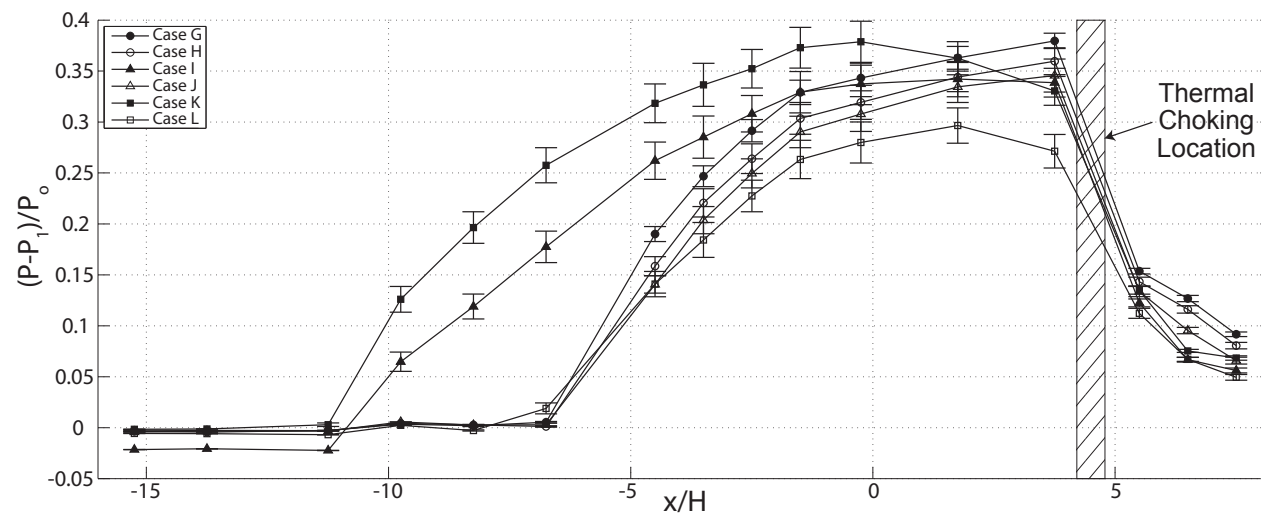

b) Pseudo-shock downstream anchor point variation

Figure 3. Pressure distribution across pseudo-shock showing a) Ram- and Scram-mode combustion and b) variation in the downstream anchor point, $x=0$ is the location of the fuel injector.

These unexpected interactions between mechanical and combustion blockages can also be seen in $3 \mathrm{~b}$ ), where six case are again provided for a set stagnation pressure, three different stagnation temperatures, $T_{o}=1000,1200 \& 1400 \mathrm{~K}$, and two equivalence ratios, $\phi=0.25 \& 0.30$. The vari- 
ation in stagnation temperature between Cases G, I \& $\mathrm{K}$ again reduces the mechanical blockage, momentum ratio, present between each case. This reduction, instead of creating a combustion mode transition as in 3a), causes a shift in the downstream anchor point of the pseudo-shock present in the isolator from the thermal throat in Case $\mathrm{G}$, forward to an intermediate position in Case I and forward again to the location of the fuel injector in Case K.

This is counterintuitive, as the point of application of the fluid-mechanical blockage would be expected to be at location of the fuel injector, with higher values causing a forward bias in the location of the maximum back pressure, and pseudo-shock anchor location. Cases H, J \& L also show this behavior to a lesser degree for a lower equivalence ratio. The geometry of the isolator and fuel injector play a key role in determining the form of the interaction between mechanical and combustion blockage.

As the effects of stagnation temperature variation on the effective blockage have already been discussed, changes to the stagnation pressure will also impact the behavior of the pseudo-shock. Figure 4a) gives longitudinal pressure profiles for a single stagnation temperature, $T_{o}=1200 \mathrm{~K}$, three different stagnation pressures, $P_{o}=310.3,448.2 \& 586.1 \mathrm{kPa}$ and two equivalence ratios, $\phi$ $=0.25 \& 0.30$. For a set equivalence ratio, an increase in stagnation pressure, Cases $\mathrm{C}, \mathrm{I} \& \mathrm{M}$, causes the pseudo-shock to grow in length, while noting the slight shift in anchor location, which is to be expected as with higher stagnation pressures comes higher static densities in the isolator flow that require a longer physical distance to be effectively compressed.

An increase in equivalence ratio is also found to result in a longer shock-train, Case I \& $\mathrm{J}$, or a combustion mode transition, Case C \& D, as previously discussed. The lengthening of the pseudoshock do to this increase in combustion blockage can be explained in terms of the associated momentum ratio increase, mechanically moving the downstream anchor point once again forward toward the fuel injection location.

The entrance to the pseudo-shock of Case $\mathrm{M}$ is actually seen to be further downstream relative to the lower stagnation pressure in Case I due to the shift in anchoring location. Case M's pseudoshock is still anchored at the thermal throat, while Case I's is anchored forward of this location due to the momentum ratio difference between these two cases. The higher stagnation pressure in Case $M$ provides a longer pseudo-shock but this is disguised by the offsetting variation in anchor location, resulting in a similar isolator length requirement but a higher density through-put at the same equivalence ratio.

Alternatively, an increase in equivalence ratio can drive an increase in the demanded maximum pressure recovered in the isolator, and required pseudo-shock length, without pushing the downstream pseudo-shock anchor point upstream, as in Figure 4b). A single stagnation condition is considered and the equivalence ratio increased. For the lowest equivalence ratios, the combined blockage, chemical plus mechanical, is insufficient to create an adequate back pressure for ram-mode operation. On increasing the equivalence ratio the combustor transitions from scram to ram-mode and the shock-train lengthens in rough proportion to the pressure recovered.

This continues until the maximum pressure recovered reaches a plateau, seen in a zero rise in the recovered pressure between Cases $\mathrm{O} \& \mathrm{~N}$ for a rise in equivalence ratio from $\phi=0.290$ to 0.301 . This limiting effect is a result of the coupling between the isolator geometry and the mechanics of the fuel injector, which will be discussed further in Section IV under the context of a behavior map coupling between isolator and fuel injector.

This limiting behavior is also observed in Figure 4a) where the stagnation pressure increase between Cases D \& $\mathbf{J}$ drives an increase in the maximum recovered pressure, while the same increase between Cases $\mathrm{C} \& \mathrm{M}$ does not, even though in absolute terms an increase in stagnation 


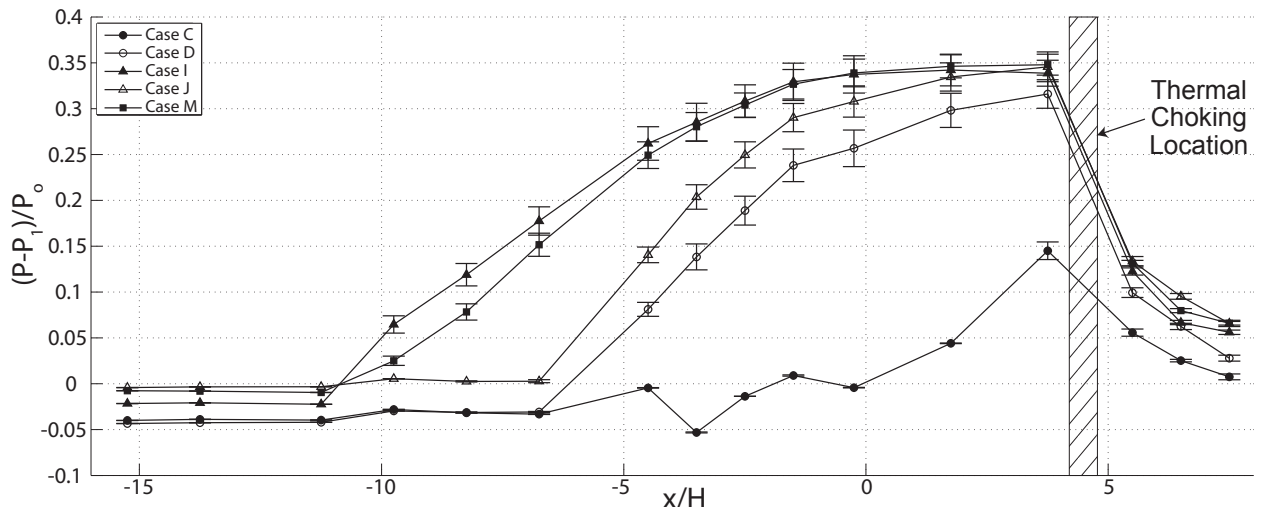

a) Stagnation pressure, $P_{o}$, dependence

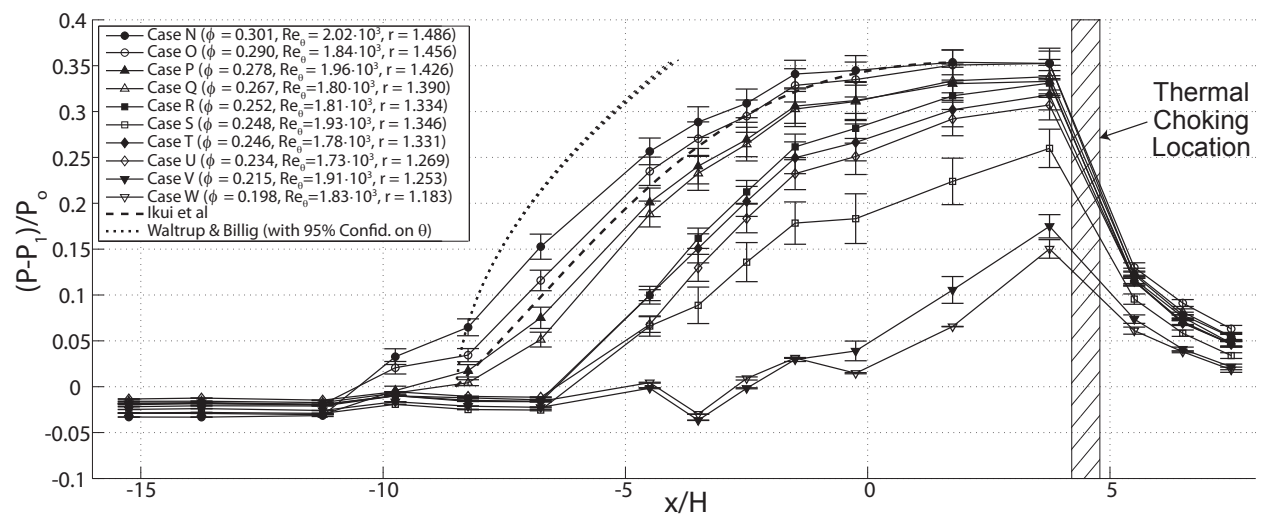

b) Equivalence ratio, $\phi$, dependence

Figure 4. Pressure distribution across pseudo-shock showing a) stagnation pressure, $P_{o}$, dependence and b) equivalence ratio, $\phi$, dependence, $x=0$ is the location of the fuel injector. 
pressure with a set equivalence ratio means a higher rate of fuel consumption and blockage.

\section{Isolator Behavior and Isolator/Combustor Coupling}

The observations that have been made to this point show a great deal of interaction between the pseudo-shock present in the isolator and the manner in which the fuel is injected into the combustor. The isolator geometry plays a key role in prescribing the pressure recovered by the isolator pseudoshock when subjected to a fuel injection induced blockage. This blockage, as previously discussed, can be attributed to one of two causes, either a chemical blockage due to the combustion of fuel or a mechanical blockage due to the fluid-mechanical obstruction created by the fuel-jet. These have been quantified here as the fuel equivalence ratio and the fuel-jet momentum ratio, respectively.

The relationship between these three parameters, for the MDMC test-section geometry, is given in Figure 5, which includes all the experimental runs undertaken in this study. The isolator pressure recovery is seen to behave as a function of equivalence ratio and fuel-jet momentum ratio, with the isolator geometry acting as the transfer function dictating the relationship between the parameters.

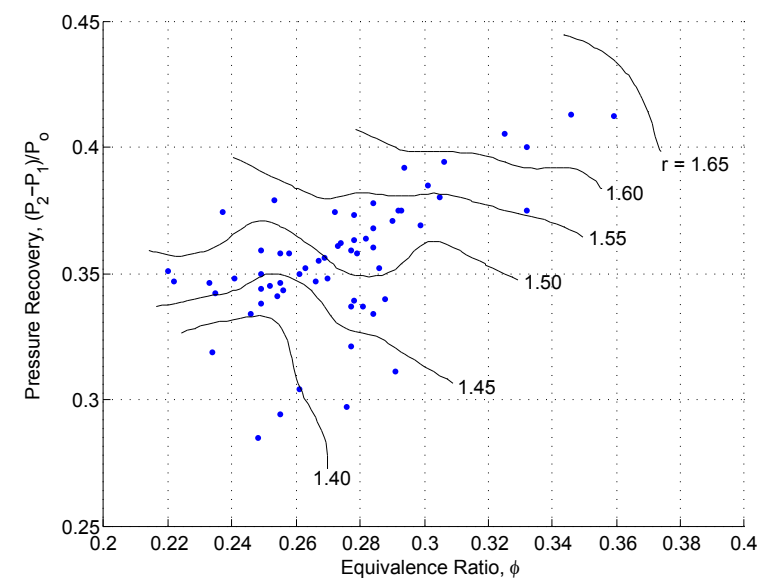

Figure 5. Isolator pressure recovery as a function of the chemical and mechanical blockage created by wallnormal fuel injection. The chemical blockage is quantified by the fuel equivalence ratio, $\phi$, and the mechanical blockage by the fuel jet momentum ratio, $r$, shown as contours. Experimental operating points are marked with dots.

In dissecting this behavior, it is instructive to examine the operation of the fuel-jet in isolation of the pseudo-shock. Considering a set of isolator stagnation conditions, $P_{o}, T_{o}$ and $M_{1}$, and an applied fuel injector pressure, $P_{F}$, will result in the injector developing one particular blockage condition, or a pairing of a fuel equivalence ratio and a fuel-jet momentum ratio. This can be seen analytically by taking Equation 2 and substituting the relation

$$
\rho u^{2}=\rho u \cdot u=\frac{\dot{m}}{A} \cdot \frac{\dot{m}}{A \rho}=\frac{\dot{m}^{2}}{A^{2} \rho}
$$

where $\dot{m}=\rho A u$ has been used, to provide

$$
r=\left[\frac{\rho_{I} A_{I}^{2}}{\rho_{F} A_{F}^{2}} \frac{\dot{m}_{F}^{2}}{\dot{m}_{I}^{2}}\right]^{1 / 2}
$$


Rearranging Equation 1 and substituting gives

$$
r=\Lambda \cdot \phi,
$$

where the injector parameter has been defined as

$$
\Lambda=\left[\left(\frac{\rho_{F}}{\rho_{I}}\right)^{-1 / 2} f_{s} Y_{O_{2}}\left(\frac{A_{F}}{A_{I}}\right)^{-1}\right] .
$$

This relation, having been developed with the assumption of a choked fuel injector orifice, must have a constant term added to account for the non-linear behavior of the injector for lower, unchocked, values of $\phi$. The injector was only run under chocked injector conditions. This constant term is a function, to first order, of the static pressures present in the combustor and the injector plenum and then Equation 5 takes the form

$$
r=\Lambda \cdot \phi+f^{n}\left(P_{2}, P_{F}\right) .
$$

This relation can be seen experimentally as well, and is shown in Figure 6 for two different stagnation pressures, where the stagnation temperature has been changed to alter the value of $\Lambda$ in each case.

This fuel injector behavior can now be overlaid onto the isolator pressure recovery map to more readily see the coupling of the pseudo-shock condition to the blockage state provided by the fuel injector, which itself is dependent on the isolator stagnation conditions and the applied fuel injector pressure. This is shown in Figure 7 where each of the fuel injector operating lines, from Figure 6, have been mapped onto the contours of fuel-jet momentum ratio from Figure 5.

Using this isolator/combustor coupling map and a known set of isolator operating conditions and fuel injector pressure, the pressure recovered in the isolator section can be easily determined. Noting again that both the blockage behavior of the fuel injector and the pressure recovery in the isolator are highly dependent on their respective geometries.

An increase in fuel injector pressure can now be considered in terms of its impact on the pressure recovery demanded from the isolator. Beginning at the operating Point " $A$ " in Figure 8, a throttle-up in fuel injector pressure will move the operating point along the fuel injector operating line to Point "B". The motion along a static operating line is reasonable so long as the throttleup occurs "fast" compared to the acceleration response time of the notional vehicle to which the engine is attached.

Once the vehicle begins to respond to the new engine output, there exist two paths that will bound the pressure recovery in the isolator and are dependent on the ordering of the change in isolator inlet conditions. If the stagnation pressure were to increases first and then the stagnation temperature, the isolator operating point will move from Point "B" to Point "E" through Point "C". If the opposite happens, with the temperature increase preceding the pressure increase, the operating point will move instead through Point "D" on its way to Point "E".

These two extreme case form the bounds for the movement of the operating point from Point "B" to Point "E", with the point always moving on a surface of constant fuel injector pressure at its intersection with the current isolator operating conditions.

\section{Boundary Layer Analysis}

In an effort to better understand the flow state present in the isolator prior to the entry of the pseudo-shock, an analysis of the boundary-layer that develops along the isolator wall was under- 


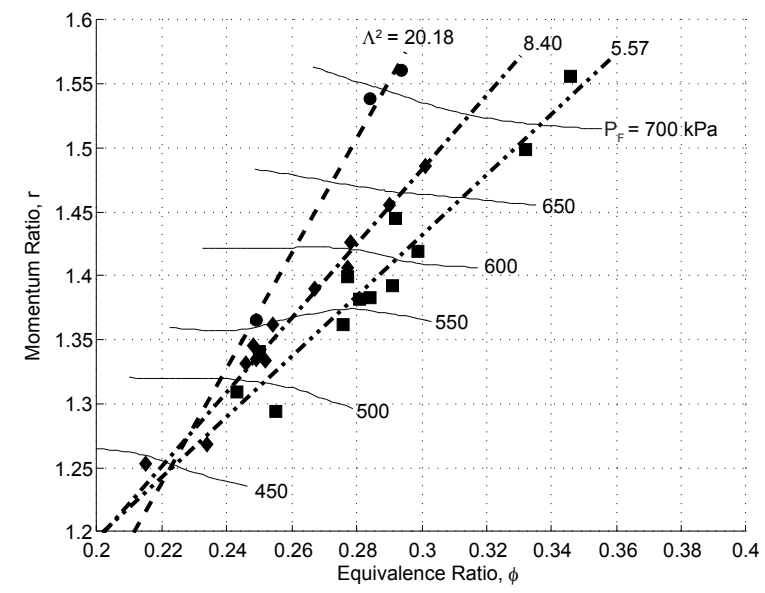

a) $P_{o}=376.2 \mathrm{kPa}$

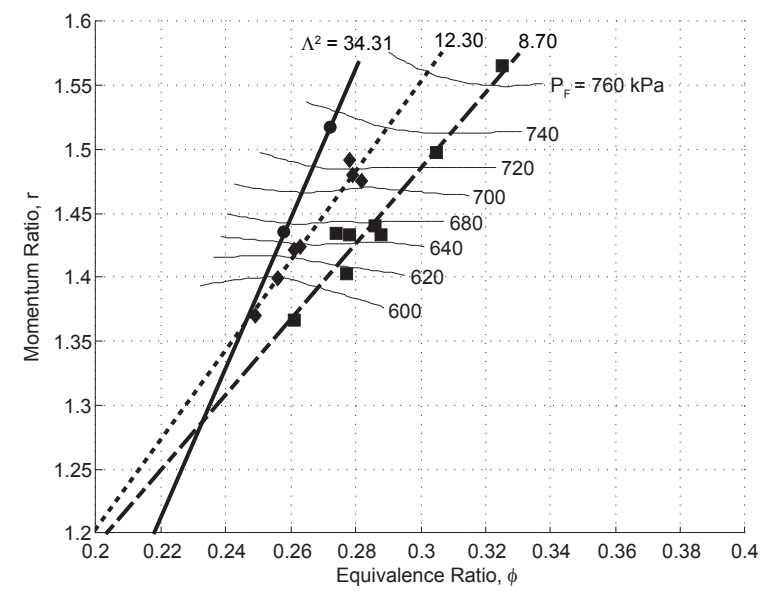

b) $P_{o}=448.2 \mathrm{kPa}$

Figure 6. Fuel injector map relating fuel equivalence ratio, $\phi$ and fuel jet momentum ratio, $r$ for a particular fuel injector geometry at an isolator flow stagnation pressure, $P_{o}$, of a) 376.2 and b) $448.2 \mathrm{kPa}$, with contours of fuel injector pressure, $P_{F}$, overlaid in $\mathrm{kPa}$. The injector parameter $\Lambda$ is given in Equation 6, with values shown for stagnation temperatures, $T_{o}$, of $1000 \mathrm{~K}, 1200 \mathrm{~K}$ and $1400 \mathrm{~K}$ moving from high to low values of $\Lambda$ respectively.

taken. Two methods were used to obtain this information. The first being a traversing stagnation Pitot pressure probe and the second Schlieren imagery of the density variations through the boundary-layer in the isolator. The second method had to be used due to the thermally induced material failure of the Pitot tube above stagnation temperatures of $T_{o}=1000 \mathrm{~K}$.

The Pitot tube arrangement consisted of a $1.19 \mathrm{~mm}$ (3/64 inch) diameter, 316 stainless steel tube, bent to allow the tip to be brought into near contact with the isolator wall, which had been attached to a computer controlled traverse to vary the position of the Pitot tube during operation of the test-section. The Mach number profiles shown in Figure 10a) were obtained from 6 separate test runs for each of the inlet stagnation pressure cases that will be presented. Much of the method used to obtain the velocity and mass-flux profiles, which are required to calculate the integral boundary-layer thicknesses, follows that outlined by Bement et al. ${ }^{3}$

The Crocco-Busemann relation is used to define a suitable static temperature profile across the 


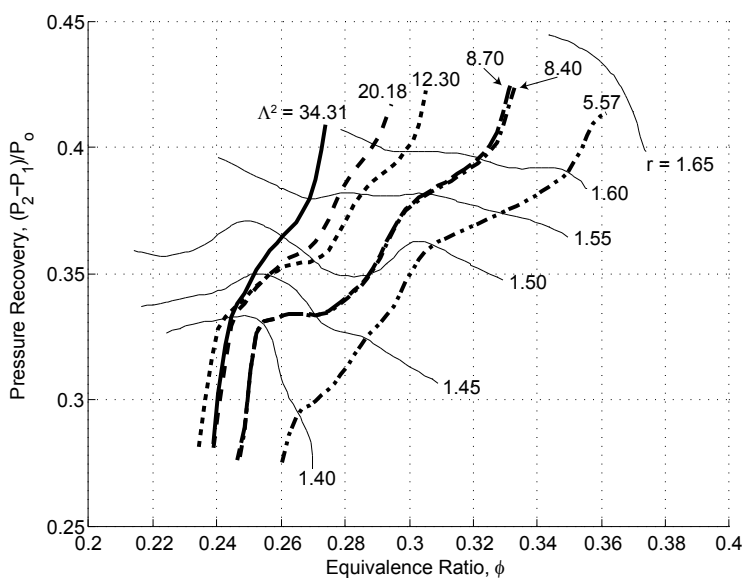

Figure 7. Isolator/Combustor coupling map showing the isolator pressure recovery, for a particular operating condition and fuel injector geometry, as a function of fuel equivalence ratio, $\phi$ and fuel-jet momentum ratio, $r$, shown as contours.

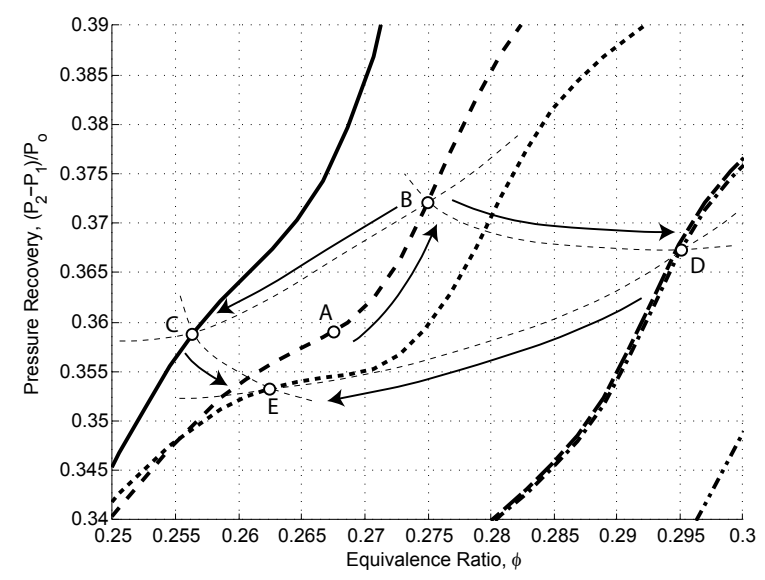

Figure 8. Isolator/Combustor coupling map showing the operating point of the isolator adjusting to an increase in fuel injector pressure. The isolator pressure recovery, for a particular operating condition and fuel injector geometry, is given as a function of fuel equivalence ratio, $\phi$ and fuel jet momentum ratio, $r$.

isolator based on the wall temperature and the local flow velocity,

$$
T=T_{w}+\frac{T_{a w}-T_{w}}{u_{e}} u-\frac{\mathfrak{r}}{2 C_{p}} u^{2}
$$

where the recovery factor, $\mathfrak{r}$, can be taken equal to $\mathrm{Pr}^{1 / 3}$. Making the appropriate substitutions for $u=M \sqrt{\gamma R T}$ and $C_{p} / R=\gamma(\gamma-1)$, the velocity distribution as a function of wall temperature and local Mach number takes the form

$$
u=\frac{M^{2} \gamma R}{2 u_{e}}\left[\frac{\left(T_{a} w-T_{w}\right)+\sqrt{\left(T_{a} w-T_{w}\right)+\frac{4 u_{e}^{2} T_{w}}{M^{2} \gamma R}\left(1+\mathfrak{r} \frac{\gamma-1}{2} M^{2}\right)}}{1+\mathfrak{r} \frac{\gamma-1}{2} M^{2}}\right]
$$

The inner wall temperature of the isolator is a required quantity in this equation but it is not easily measured, particularly without introducing an excess of disturbances into the flow. To address 
this, a unsteady finite-difference model was constructed to model the evolution of the temperature over the length of a test-section run. The model results for a number of test conditions were evaluated by comparing the exterior test-section wall temperature indicated in the model to the actual temperature obtained from infra-red thermometer measurements taken during operation of the test-section. They were found to agree to within $8.5 \%$ and as such there is confidence that the convective and thermal diffusion effects present were being modeled in an appropriate manner to provide a reliable estimation of the isolator inner wall temperature.

The boundary-layer profiles for the inlet stagnation pressures of $310.3,448.2 \& 586.1 \mathrm{kPa}$ and a stagnation temperature of $1000 \mathrm{~K}$ are compiled in Figure 10. As only the lowest stagnation temperature being considered was actual measurable, the Schlieren method was then used to provide estimates at all stagnation conditions that could be compared to the more accurate Pitot measurements of the integrated boundary-layer quantities.

Schlieren images of the flow were taken for all 9 test conditions being considered, combinations of the stagnation pressures of $310.3,448.2 \& 586.1 \mathrm{kPa}$ and stagnation temperatures of 1000,1200 \& $1400 \mathrm{~K}$. The light amplitude variations created by the Schlieren technique are due to gradients in the index-of-refraction, $n$, of the gas inside the test-section, through which the light rays have passed. These gradients are then proportional to the angular light ray deflection by

$$
\epsilon_{x}=\frac{L}{n_{o}} \frac{\partial n}{\partial x}, \quad \epsilon_{y}=\frac{L}{n_{o}} \frac{\partial n}{\partial y},
$$

where $L$ is the length of the Schlieren media and $n_{o}$ the base index-of-refraction of the gas. These deflection angles are related to the gas density gradients by $n-1=k \rho$, with $k$ being the GladstoneDale Coefficient for the gas $\left(\approx 0.23 \mathrm{~cm}^{3} / \mathrm{g}\right.$ for air in visible light $)$. The image intensity contrast can then be related back to the density gradients via these deflection angles,

$$
\frac{\Delta E}{E} \propto \epsilon_{y} \propto \frac{\partial \rho}{\partial y}
$$

assuming that the knife-edge cut-off has be oriented parallel to the flow direction. The reader is referred to Settles book on this technique for a more complete description. ${ }^{8}$

The gradient in density is also proportional to the gradient in static temperature through the ideal gas law, and a relative measure of the thermal boundary-layer profile is now attainable. The first quantity that must be identified form this gradient profile is the thickness of the velocity boundary-layer. This can be done by identifying the thickness of the thermal boundary-layer from the Schlieren images, that have been collapsed from a two-dimensional image to a one-dimensional profile of image intensity. As can be seen in Figure 10c), this point can be identified by the change in the gradient of the temperature profile, or rather the image contrast information as they are proportional.

The thermal boundary-layer thickness can now be adjusting in the usual may, via the Prandtl number, to obtain an estimate of the velocity boundary-layer thickness. The rest of the image contrast information, between the thermal boundary-layer thickness point and the wall is used to estimate the profile of the velocity distribution in a similar manner.

The integral boundary-layer thicknesses could then be calculated numerically for all instances from

$$
\delta^{*}=\int_{0}^{\delta}\left(1-\frac{\rho u}{\rho_{e} u_{e}}\right) d y
$$


and

$$
\theta=\int_{0}^{\delta} \frac{\rho u}{\rho_{e} u_{e}}\left(1-\frac{u}{u_{e}}\right) d y,
$$

the results of which are shown in Figure 9. As the figure shows, the Schlieren results for boundarylayer velocity and momentum thicknesses agree well with those obtained from the Pitot tube, while those for displacement thickness do not. This will be due to the inability of the Schlieren method of adequately recreate the boundary-layer velocity profile in the wall-normal direction. The velocity thickness estimation does not rely on this profile but is directly read from the thermal boundarylayers impact on the density gradients in the flow. The momentum thickness, while it is dependent on the velocity profile, is less sensitive to inaccuracies than the displacement thickness due to the fact this is a supersonic flow and the majority of the momentum carried within the boundary-layer will be in the portion furthest form the wall, which has been shown to be estimated reliably.

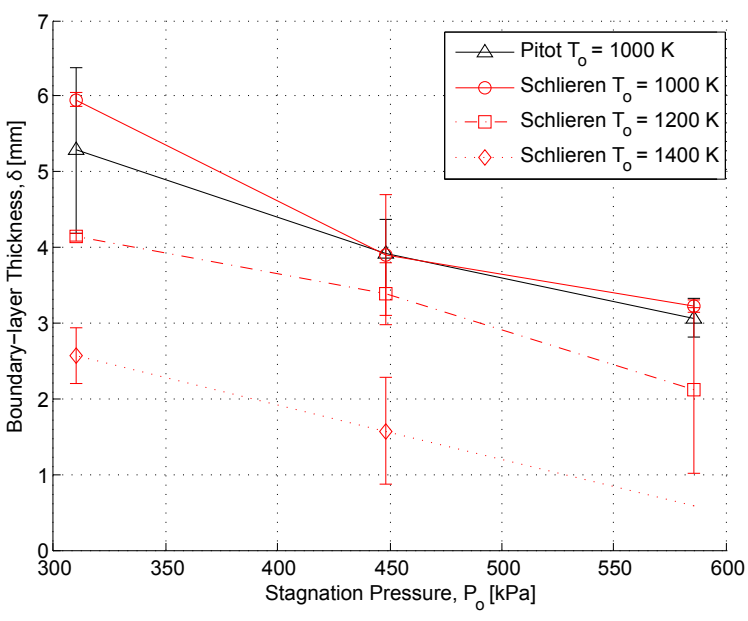

a) Boundary-layer thickness, $\delta_{99 \%}$.

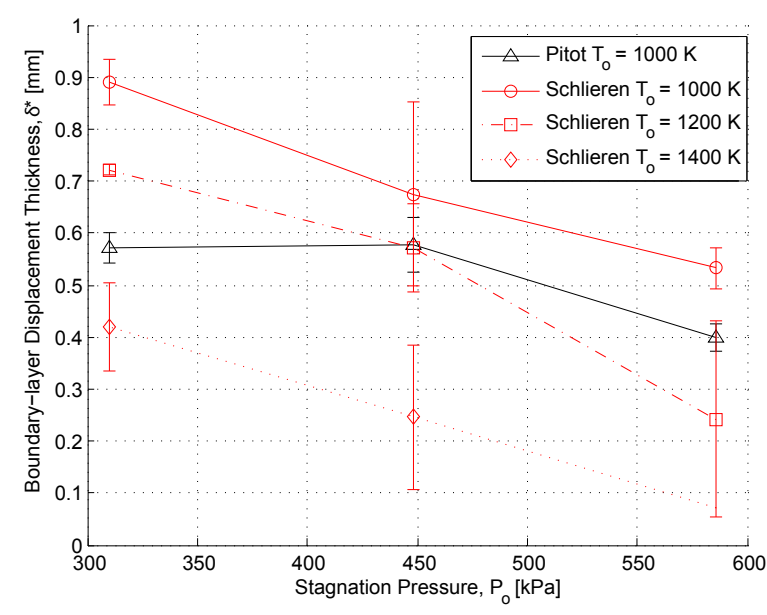

b) Boundary-layer displacement thickness, $\delta^{*}$.

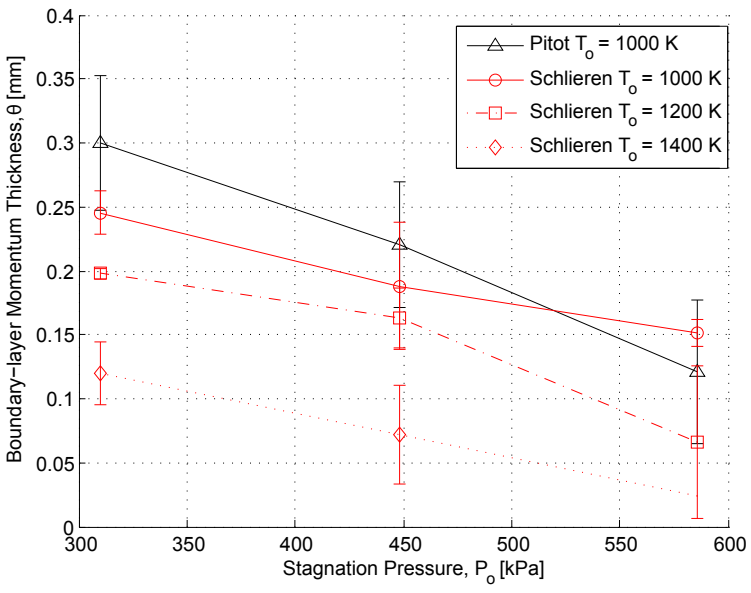

c) Boundary-layer momentum thickness, $\theta$.

Figure 9. Boundary-layer thickness measurements based on boundary-layer profiles obtained through Pitot tube and Schlieren techniques. 


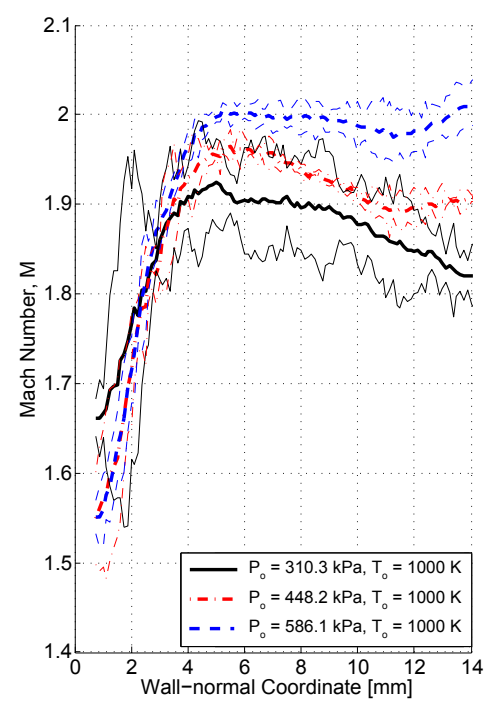

a)

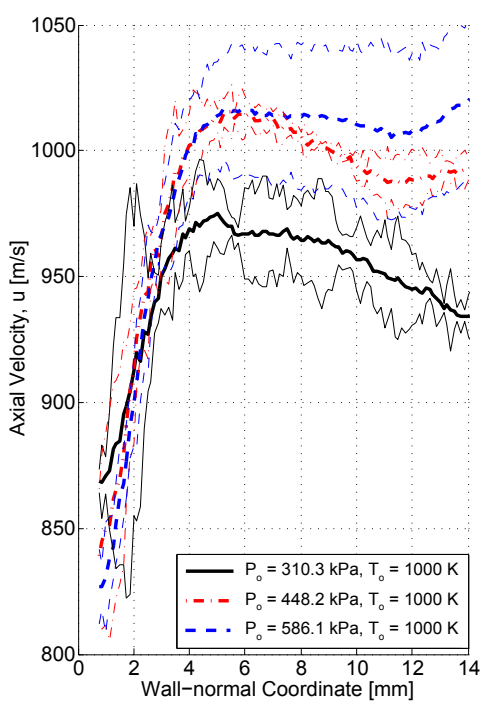

b)

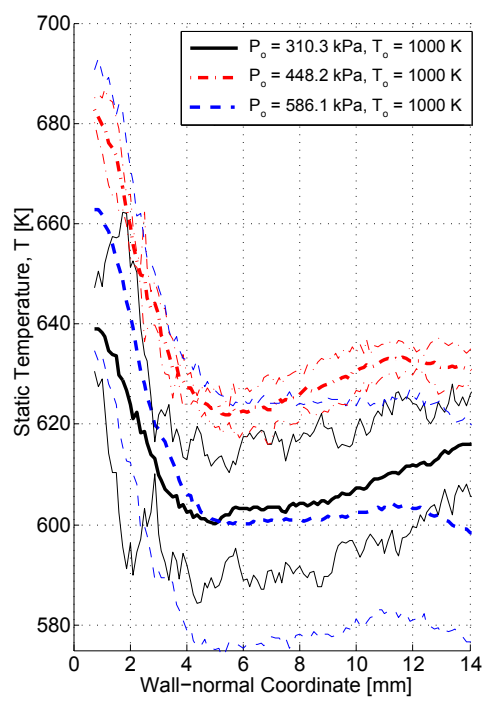

c)

Figure 10. Boundary-layer profiles of a) Mach number, b) axial velocity and c) static temperature as measured using a Pitot stagnation pressure probe. Measurement error bounds are given as light-weight lines of similar format.

\section{Pseudo-shock Pressure Rise \& Length}

For each of the experimental cases, pressure measurements have been made along the length of the isolator section. This allows for a comparison to be made with existing models of the pressure rise that occurs across a pseudo-shock. Models have been proposed beginning with Crocco's ${ }^{9}$ shockless model which assumes that the presence of the shocks can be disregarded entirely with the dissipation in the flow being due to the turbulence in the near wall regions. A uniform and isentropic central core flow is also assumed, giving

$$
\frac{P}{P_{1}}=\left(\frac{1-w^{2}}{1-w_{1}^{2}}\right)^{\frac{\gamma}{\gamma-1}}
$$

where $w^{\prime}$ is the Crocco number of the supersonic core. Quantities with a subscript 1 refer to the pre-pseudo-shock flow state and those with a 2 to the post-pseudo-shock state.

The diffusion model of Ikui et $\mathrm{al}^{10}$ improves on this relation by considering that the central core region in the duct is not isentropic, allowing for the pressure rise across a pseudo-shock to take the form

$$
\frac{P-P_{1}}{P_{2}-P_{1}}=\frac{\left\{w_{1}^{2}\left(w_{1}^{2}-2 w^{* 2}\right)+w_{1}^{2} w^{* 2} e^{-c(x / D)}\right\}\left(1-e^{-c(x / D)}\right)}{\left(w_{1}^{2}-w^{* 2}\right)^{2}-w_{1}^{2}\left(w_{1}^{2}-w^{* 2}\right) e^{-c(x / D)}\left(1-e^{-c(x / D)}\right)},
$$

with $c=0.114$ being determined from experiment. A separate relation for the length of the pseudo-shock, $L_{p}$, is provided using the same assumptions,

$$
\frac{L_{p}}{D}=\frac{2}{c} \sinh ^{-1}\left(\frac{w_{1}-w_{2}}{2 w^{*}}\right)
$$

It should be noted that this equation is not predictive of the maximum pressure recovered, but describes the profile of the pressure rise once both the pre- and post-pseudo-shock states are known. 
The later modified diffusion model of Ikui et al ${ }^{11}$ takes into account the friction losses to the walls of the duct, as well as the effect of the upstream boundary layer. Zimont and Ostras ${ }^{12}$ also proposed a model which assumes that the dissipation inside the pseudo-shock takes on a jet-like structure and that it behaves in the manner of a submerged supersonic jet.

Waltrup and Billig ${ }^{4,5}$ offer the following correlation to experimental data obtained for a cylindrical duct:

$$
\frac{x\left(M_{1}^{2}-1\right) R e_{\theta}^{1 / 4}}{D^{1 / 2} \theta^{1 / 2}}=50\left(\frac{P}{P_{1}}-1\right)+170\left(\frac{P}{P_{1}}-1\right)^{2},
$$

where $\theta$ is the momentum thickness of the undisturbed, pre-shock train, boundary-layer and $R e_{\theta}$ is the Reynolds number of the flow based on this length scale.

The preceding analysis of the boundary-layers that are present under each of the experimental conditions can now be used with these models to asses their accuracy, and applicability, in estimating the key parameters of pseudo-shock length and pressure recovery.

Waltrup and Billig's correlation and Ikui et al's diffusion model are compared to experimental results to asses their ability to predict the longitudinal pressure rise profile along the isolator for instances where the stagnation temperature, Figure 11a), and stagnation pressure, Figure 11b), have been varied, with the particular model parameters adjusted accordingly. The behavior of the correlation was found to be more sensitive to the estimated Reynolds number then the momentum thickness of the boundary-layer itself.

Ikui et al's diffusion model is found to provide fair agreement with the experimental profiles, showing some underestimation at the beginning of the pseudo-shock. As Bement at al found for their results, Waltrup and Billig's correlation was also found to agree well with the experimental data, however its agreement seems to be highly dependent on the Reynolds number of the flow being considered. Case $\mathrm{M}$ in Figure 11b) shows this well, as the rate of pressure rise is overpredicted. Case $\mathrm{M}$ has a $R e_{\theta}=0.96 \cdot 10^{3}$ placing it just outside the lower limits of the data considered by Waltrup and Billig in fitting their data. ${ }^{4}$ Case G, shown in Figure 11a), also has the potential to show this behavior, but the slightly higher Mach number of the flow as compared to Case $\mathrm{M}$ compensates for some of the over-prediction.

This tendency to over-predict the pressure rise for lower momentum thickness Reynolds number flows must be highlighted, as this quantity is very sensitive to the isolator cross-sectional geometry. This can be seen from the values reported by Waltrup and Billig ${ }^{4}$ and Bement. ${ }^{3}$ The later for instance took data using the Generic High Speed Engine model at NASA/Langley, which while operated at comparable pressures, temperatures and Mach number, had a physically larger isolator cross-section of $101.6 \mathrm{~mm}$ x $104.6 \mathrm{~mm}$ (compared to the MDMC isolator at $25.4 \mathrm{~mm} \times 38.1 \mathrm{~mm}$ ), providing thicker boundary-layers.

This has implications on the size of the system, wave-rider as compared to missile-scale, that this correlation is being used to describe. Care should be taken to ensure that the momentumthickness Reynolds number of the flow falls within those considered by Waltrup and Billig in creating their fit, to ensure proper, applicable, predictions of the pseudo-shock's behavior.

These limits on applicability in mind, a comparison of the more macroscopic quantity of overall pseudo-shock length can be made between these models, incorporating the influences of momentum and equivalence ratio on the blockage encountered by the isolator flow, as described earlier. Figure 12 provides a comparison between Ikui et al's diffusion model, Waltrup and Billig's experimental correlation and experimental data arranged by two equivalence ratios, but over a variation in momentum ratio for each. In both cases, Ikui et al's pseudo-shock length prediction is found to be 


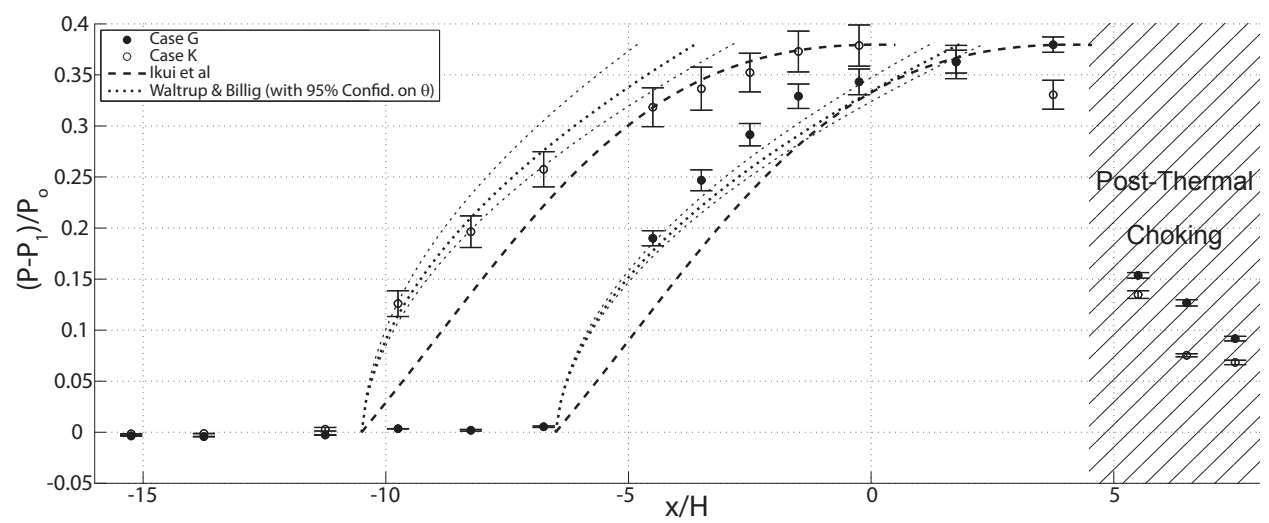

a) Pseudo-shock approximation with stagnation temperature, $T_{o}$, dependence.

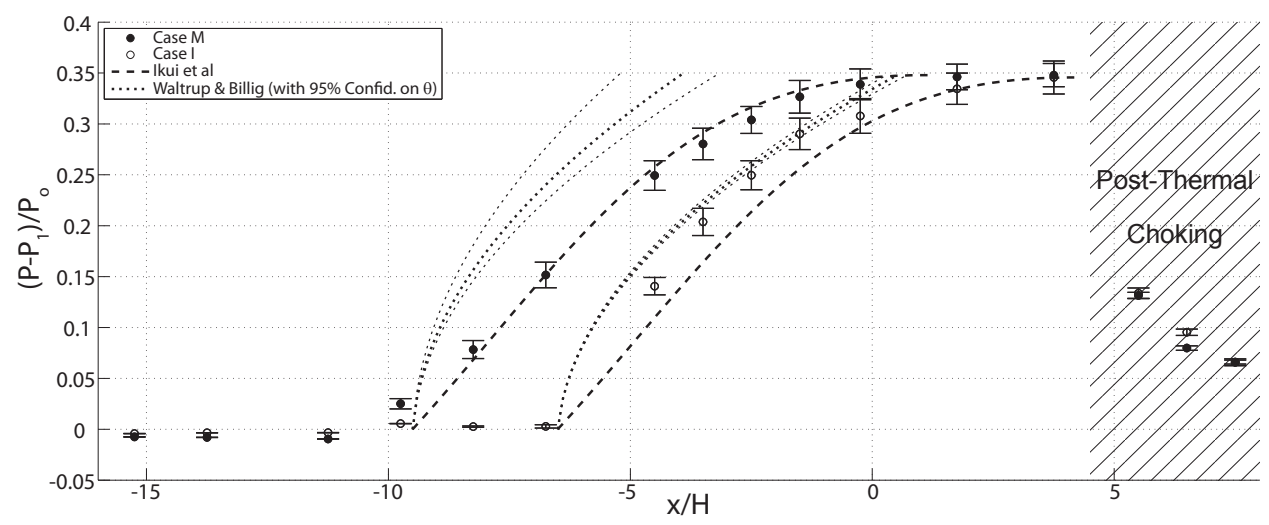

b) Pseudo-shock approximation with stagnation pressure, $P_{o}$, dependence.

Figure 11. Pressure distribution across pseudo-shock showing a) stagnation temperature, $T_{o}$, dependence and b) stagnation pressure, $P_{o}$, dependence in comparison to the diffusion model of Ikui et $\mathrm{al}^{10}$ and the experimental correlation of Waltrup and Billig, ${ }^{4} x=0$ is the location of the fuel injector. 
an overestimation, while Waltrup and Billig's an underestimation with the earlier noted constraints in mind.

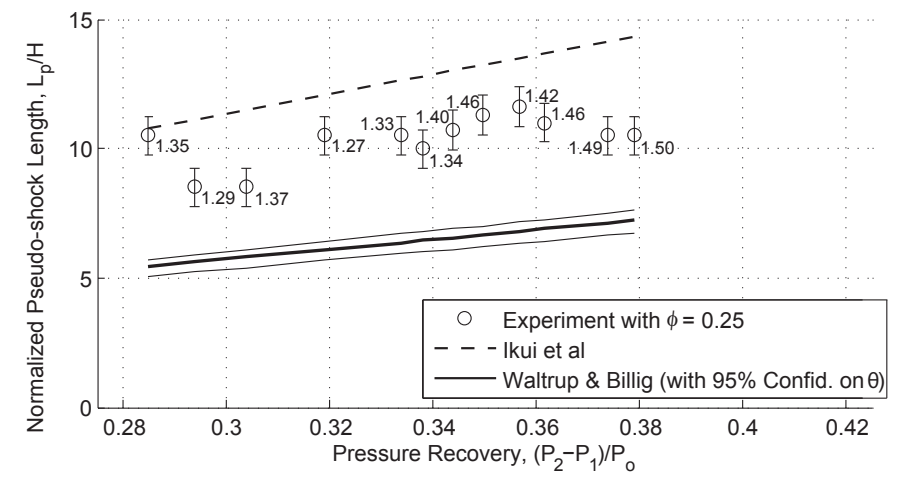

a) $\phi=0.25$

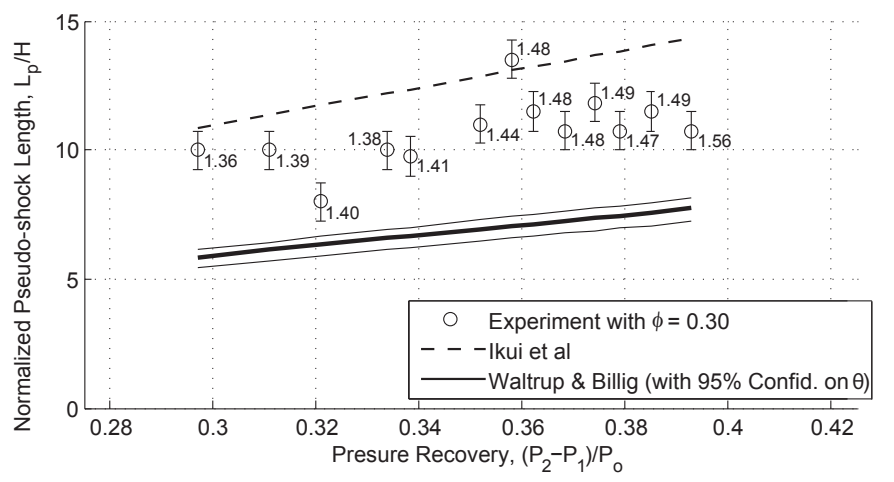

b) $\phi=0.30$

Figure 12. Comparison of predicted pseudo-shock length from experiment for equivalence ratio, $\phi$, of a) 0.25 and b) 0.30 with the diffusion model of Ikui et $\mathrm{al}^{10}$ and the experimental correlation of Waltrup and Billig ${ }^{4}$ as a function of Maximum Pressure Recovery, $\left(P_{2}-P_{1}\right) / P_{o}$. Labeled values are the momentum ratio, $r$, for each given condition.

It can be seen in both instances that the momentum ratio plays an important roll in driving both an increase in the length of the pseudo-shock and the maximum pressure it will recover over that length. This reinforces the point that the mechanical blockage created by the fuel-jet must be examined in the implementation of any injector design, in particular when considering the use of supersonic injectors or angled injectors that will increase or decrease, respectively, the wall-normal momentum ratio provided by a particular design.

\section{Shock-train Structure \& Operating Regimes}

To aide in the interpretation of the pressure field measurements, Schlieren images were obtained of the shock train present in the isolator duct. These images allowed for the structure of the pseudo-shock to be examined and the regime under which the isolator shock-train was operating to be identified. These operating regimes include the existence of a simple normal shock, a curved shock, a branched normal shock or a series of shocks. A good discussion of these structures is presented by Matsuo et al, ${ }^{1}$ in which the occurrence of a particular operating regime is linked to the flow confinement, or the ratio of the undisturbed boundary-layer thickness to the channel 
half-height $\delta / h$, and the isolator inlet Mach number, $M_{1}$.

Carrol and Dutton ${ }^{13}$ outline that for small values of $\delta / h$ a normal shock occurs, a series of nearly normal shocks for moderate values of $\delta / h$ and a series of oblique shocks for large $\delta / h$. The discrimination between small and large flow confinement values appears in the literature to be fairly qualitative, with the inlet Mach number influencing this definition. An increase in inlet Mach number effectively decreases the flow confinement, hastening the onset of the full shocktrain structure, while increasing the spacing between shocks already in series. This shock-train regime description, while subjective, has been found to generally describe the observed trends.

These shock-train regimes are shown in Figure 13, with frames of Schlieren imagery from the MDMC isolator section, taken at $100 \mathrm{~Hz}$. The others regimes are notionally depicted where unobservable due to the physical arrangement of the test-section. There are deviations from the established shock-train regime description that need to be addressed.

Having developed an estimation of the undisturbed boundary-layer thickness for the conditions shown, the inversion of the regimes with regard to the effects of flow confinement should be noted. The observed flows are placed in the regime containing a series of normal shocks if the conventional description is followed. ${ }^{13}$

In the experimental cases examined here, the isolator inlet Mach number is nominally set at 2.2, under which conditions the pseudo-shock should have marginally entered into the full oblique shock-train regime, see Figure 13e), that has been previous observed to occur for Mach numbers larger than 1.8-2.2. ${ }^{1}$

These two conflicting observations highlight a need to better understand the physical inter-play between the normal shock and oblique shock-train regimes at lower values of flow confinement. During efforts to better understand this inconsistency, a mechanism for the breakdown of the normal shock-train and transition to an oblique shock-train has been observed, and is shown in Figure 14 in consecutive Schlieren images taken at $100 \mathrm{~Hz}$. The steps in the breakdown mechanism are outlined in Figure 15, where frame references to Figure 14 are given as support for the arguments.

The mechanism begins when oblique shocks of a sufficient strength are created by the impingement of the pre-shock-train boundary-layer into the flow. The height of the leading normal shock in the train is reduced by its intersection with these confinement induced oblique shocks.

A point will be reached when the oblique shocks have strengthened to the extent that the flow now entering the leading normal shock in insufficient to allow the normal shocks continued stable existence. The leading normal shock is then found to collapse upon itself, forming a cluster of intersecting oblique shocks who's lifetime is dependent on the flow confinement present in the isolator. If the confinement is insufficient to perpetuate the oblique shock structure a leading normal shock will reform, followed by a surge forward in the position of the entire shock-train in the isolator. After the re-establishment of the leading normal shock a pair of oblique shocks are found to cross just upstream of this location. The significance of this formation is under examination.

The frequency of this cycle, and the oblique shock lifetime, was found to increase not only for instances of increased flow confinement, but also for cases demanding higher back pressures, or pressure recovery, from the shock-train. An increased pressure rise across the leading normal shock, or a stronger shock, will create an thicker post-shock boundary-layer thickness. ${ }^{14}$ This inturn will weaken the normal shock at its upper and lower edges, allowing a weaker oblique shock to initiate the normal-to-oblique shock-train transition.

A single mechanism can now be used to describe the inconsistencies in shock-train regime outlined earlier, as well as the established effects of flow confinement observed by others. Figure 16 gives a shock-train regime diagram incorporating these new effects. On the right of the diagram

19 of 24

American Institute of Aeronautics and Astronautics 


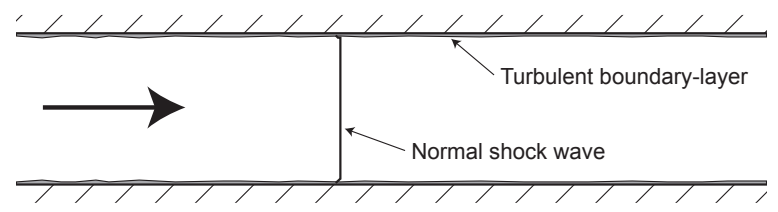

a) Single normal shock at small $\delta / h$.

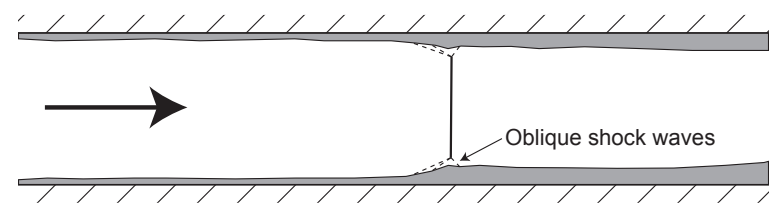

b) Single branched-normal shock moderate $\delta / h$.
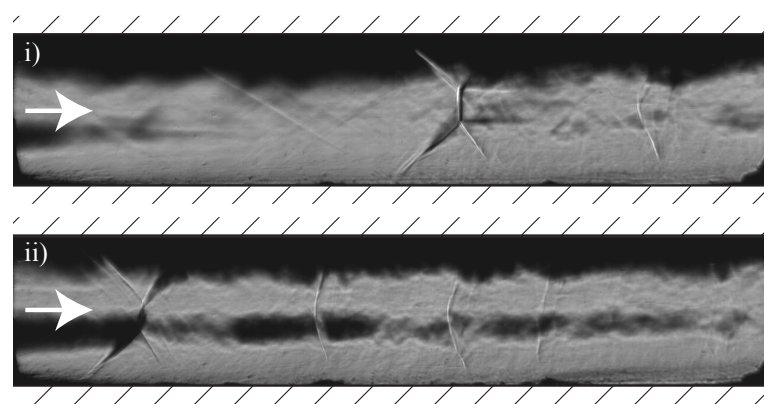

c) Branched shock followed by bowed normal shocks $L_{p}=279.4-305 \mathrm{~mm}$ and $\delta / h=0.224-0.302$.
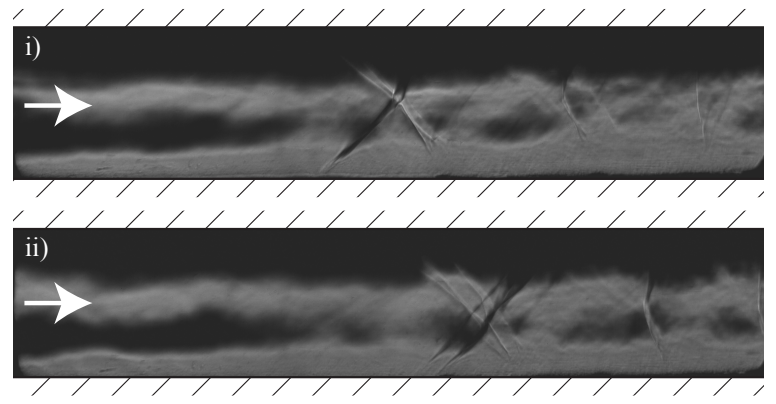

d) Oblique shocks followed by bowed normal shocks $L_{p}=280 \mathrm{~mm}$ and $\delta / h=0.121-0.122$.

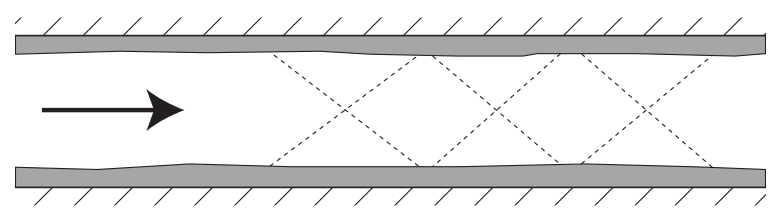

e) Oblique shocks for large $\delta / h$.

Figure 13. Shock-train stucture regimes possible in the constant area isolator section, for various run conditions. 

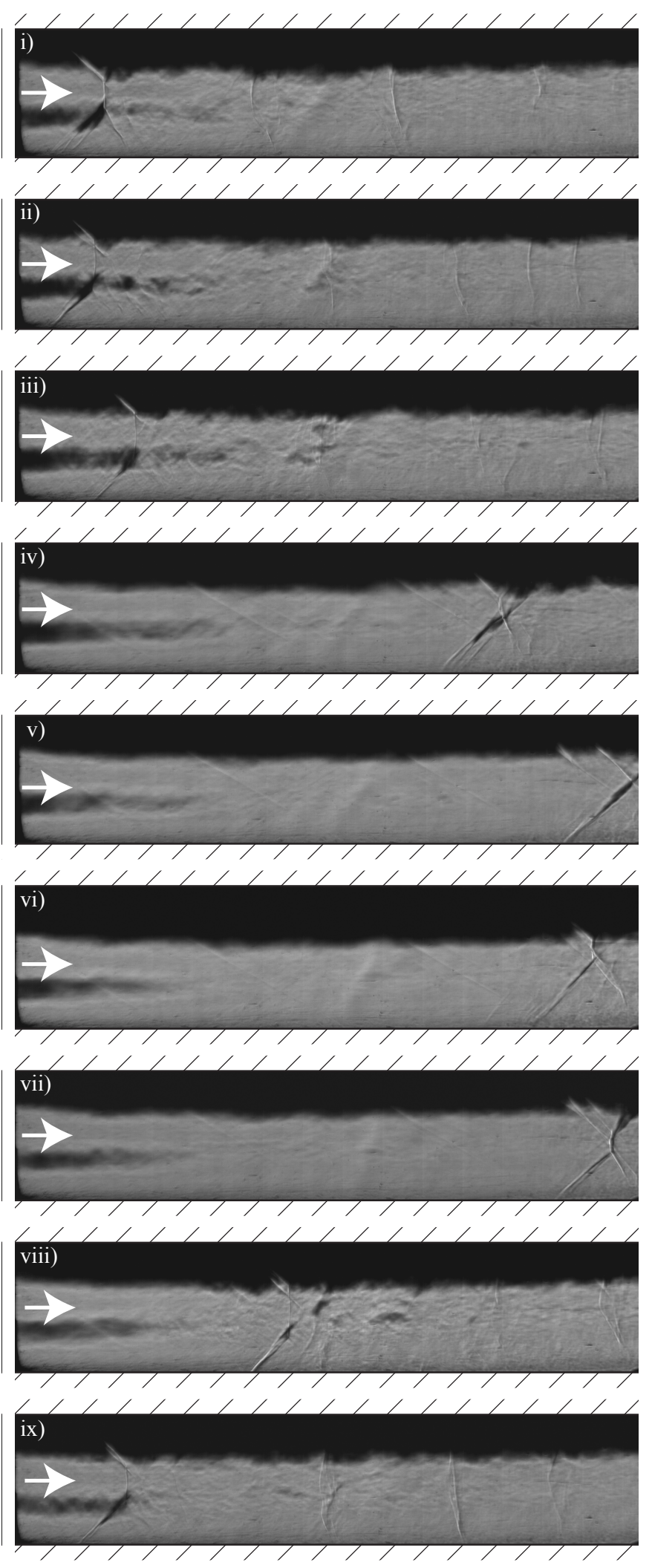

Figure 14. Proposed normal shock-train breakdown mechanism, shown in Schlieren imagery taken at $100 \mathrm{~Hz}$, with knife-edge parallel to the flow, for a stagnation pressure of $448.2 \mathrm{kPa}$ and temperature of $1200 \mathrm{~K}$. 


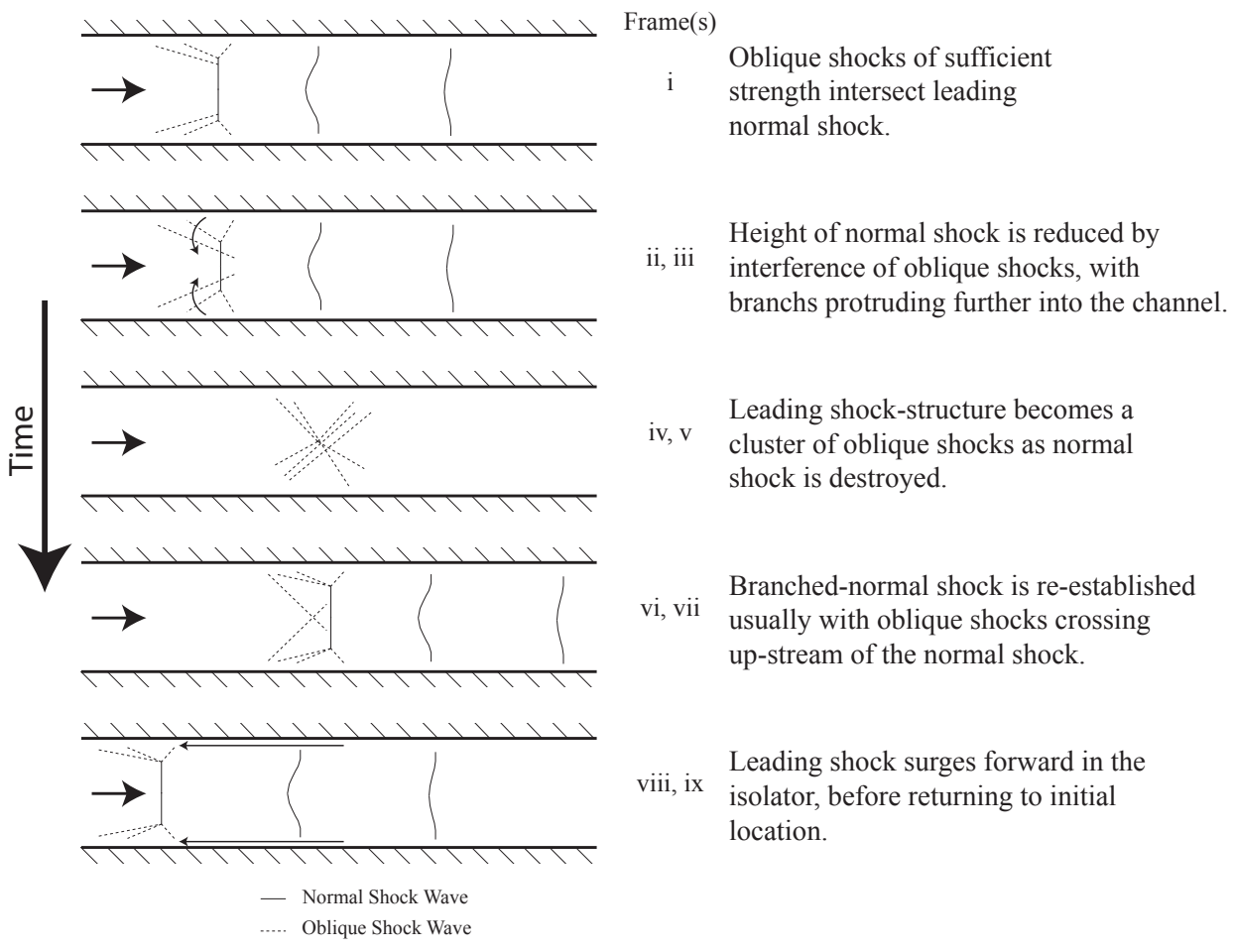

Figure 15. Proposed normal shock-train breakdown mechanism, shown in illustration, with references to Schlieren image frames in Figure 14.

the established influence of flow confinement has be hatched. This represents the impact of the pre-shock-train boundary-layer on creation of oblique shocks, and has been labeled as the Oblique Shock Confinement Limit on the normal shock-train.

For intermediate values of flow confinement, the shock-train regime will be dictated by isolator inlet Mach number, the inlet stagnation conditions, and the pressure recovery demanded by the downstream blockage. All of these parameters influence the length of the pseudo-shock present in the isolator and as such this is what has been used to quantify these effects vertically on the regime diagram.

The kink in the steady normal shock-train boundary with the region denoting the presence of the transition mechanism shows the balance point between a compressive efficiency dictated regime and the shock-train length dictated regime for intermediate values of flow confinement. The longer a pseudo-shock becomes the more viable the existence of oblique shocks to provide the required compression.

At very low values of flow confinement and very short pseudo-shock lengths, the only shock structure that can be permitted is a single normal shock. The need of efficiency in compressing the flow in a short length requires this Normal Shock Compression Limit, and is consistent again with the established regime delineation.

\section{Summary}

The behavior of the pseudo-shock created in a supersonic isolator by a hydrogen-air combustor has been studied for the instances of heated inlet flows up to $1400 \mathrm{~K}$. The need to consider 


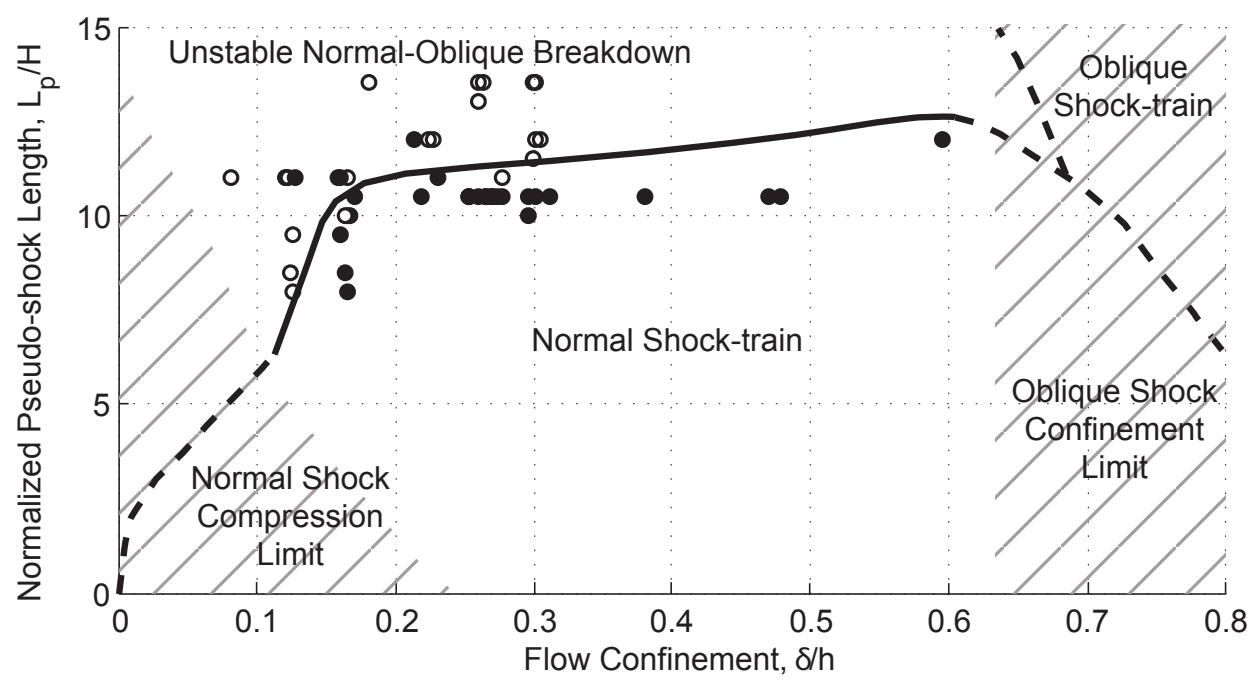

Figure 16. Pseudo-shock regime as a function of the flow confinement, $\delta / h$, and the normalized pseudo-shock length, $L_{p} / H$. Observed stable normal shock-train behavior is shown as closed dots, and unstable behavior as open dots.

the effects of both the combustion and mechanically induced blockage in the combustor has been identified through its influence on downstream pseudo-shock anchor point and the maximum pressure recovery demanded of the isolator. Fuel equivalence ratio and fuel-jet momentum ratio having been used to quantify these two coupled blockage parameters, respectively.

The fuel injector developed blockage, and for instance its influence on the maximum pressure recovery, was found to play a role in dictating the operating regime of the shock-train, requiring a reconsideration of the established shock-train regime delineation. The flow confinement, $\delta / h$, coupled with the normalized pseudo-shock length, $L_{p} / H$, have been used to describe this new operating space. As well, a normal shock-train breakdown and transition to oblique shock-train mechanism has been experimentally observed and its role in moving between discrete structural regions in the new regime description outlined.

These findings led to the consideration of the coupling between the isolator and fuel injector. Mappings were developed which show the relation between the fuel equivalence ratio and fueljet momentum ratio, and the pressure recovered in the isolator. Injector behavior at particular operating conditions are then overlaid to give a methodology for representing the intricate effects of isolator geometry, injector design and operating condition on a single coupling map. This isolator/injector mapping can then be used to predict the operation of the system for given operating conditions.

\section{References}

\footnotetext{
${ }^{1}$ Matsuo, K., Miyazato, Y., and Kim, H.-D., "Shock Train and Pseudo-shock Phenomena in Internal Gas Flows," Progress in Aerospace Sciences, 1999.

${ }^{2}$ Nill, L. D. and Mattick, A. T., "An Experimental Study of Weak Normal Shock-wave/Turbulent Boundarylayer Interactions in Internal Flows," AIAA 34th Aerospace Sciences Meeting and Exhibit, 1996, p. 2, AIAA Paper NO. 96-0799.

${ }^{3}$ Bement, D. A., Stevens, J. R., and Thompson, M. W., "Measured Operation Characteristics of a Rectangular Combustor/Inlet Isolator," AIAA/SAE/ASME/ASEE 26th Joint Propulsion Conference, 1990, p. 2, AIAA Paper No.
} 
90-2221.

${ }^{4}$ Waltrup, P. J. and Billig, F. S., "Structure of Shock Waves in Cylindrical Ducts," AIAA Journal, Vol. 11, No. 10, 1973, pp. 1404-08.

${ }^{5}$ Waltrup, P. J. and Billig, F. S., "Prediction of Precombustion Wall Pressure Distributions in Scramjet Engines," Journal of Spacecraft, Vol. 10, No. 9, 1973, pp. 620-22.

${ }^{6}$ D B Le, C P Goyne, R. H. K. and McDaniel, J. C., "Experimental Study of a Dual-Mode Scramjet Isolator," Journal of Propulsion and Power, Vol. 24, No. 95, 2008, pp. 1050-57.

${ }^{7}$ Micka, D. J. and Driscoll, J. F., "Combustion Characteristics of a Dual-Mode Scramjet Combustor with Cavity Falameholder," Proceedings of the Combustion Institute, Vol. 32, 2009, pp. 2397-2404.

${ }^{8}$ Settles, G., Schlieren and Shadowgraph Techniques, Experimental Fluid Mechanics, Springer, 1st ed., 2001.

${ }^{9}$ Crocco, L., One-Dimensional Treatment of Steady Gas Dynamics, Princeton University Press, 1958, pp. 11030.

${ }^{10}$ Ikui, T., Matsuo, K., and Nagai, M., "The Mechanism of Pseudo-Shock Waves," Bulletin of the JSME, 1974.

${ }^{11}$ Ikui, T., Matsuo, K., and Sasaguchi, K., "Modified Diffusion Model of Pseudo-shock Waves Considering Upstream Boundary Layers," Bulletin of the JSME, 1981.

${ }^{12}$ Zimont, V. L. and Ostras, V. N., "Calculation of Pseudo-shocks in a Cylindrical Duct," Fluid Mec Soviet Res, Vol. 5, No. 2, 1979, pp. 78-87.

${ }^{13}$ Carroll, B. F. and Dutton, J. C., "Characteristics of Multiple Shock Wave/Turbulent Boundary-Layer Interactions in Rectangular Ducts," Journal of Propulsion, 1989.

${ }^{14}$ Shapiro, A. H., The Dynamics and Thermodynamics of Compressible Flow, Wiley, 1975. 